\title{
A Pommaret Bases Approach to the Degree of a Polynomial Ideal
}

\author{
Bentolhoda Binaei · Amir Hashemi · \\ Werner M. Seiler
}

Received: / Accepted: date

\begin{abstract}
In this paper, we study first the relationship between Pommaret bases and Hilbert series. Given a finite Pommaret basis, we derive new explicit formulas for the Hilbert series and for the degree of the ideal generated by it which exhibit more clearly the influence of each generator. Then we establish a new dimension depending Bézout bound for the degree and use it to obtain a dimension depending bound for the ideal membership problem.
\end{abstract}

Keywords Polynomial ideals · Gröbner bases · Pommaret bases · Quasi stable ideals · Hilbert series · Degree of ideals · Bézout's bound

Mathematics Subject Classification (2000) 13P10 14 Q20

\section{Introduction}

Gröbner bases, introduced by Bruno Buchberger in his $\mathrm{PhD}$ thesis (see [10, 11]), have become a powerful tool for constructive problems in polynomial ideal theory and related domains. It is well-known that they allow us to design algorithms for computing important invariants like dimension, degree and Hilbert function. However, the bases themselves are largely independent of the

Bentolhoda Binaei

Department of Mathematical Sciences, Isfahan University of Technology

Isfahan, 84156-83111, Iran

E-mail: H.Binaei@math.iut.ac.ir

Amir Hashemi

Department of Mathematical Sciences, Isfahan University of Technology

Isfahan, 84156-83111, Iran;

E-mail: Amir.Hashemi@cc.iut.ac.ir

Werner M. Seiler

Institut für Mathematik, Universität Kassel

Heinrich-Plett-Straße 40, 34132 Kassel, Germany

E-mail: Seiler@mathematik.uni-kassel.de 
values of these invariants. This is in marked contrast to Pommaret bases which reflect many combinatorial and homological properties of the ideals they generate. They are not only of computational interest by providing easy access to many invariants relevant for algebraic geometry, but also allow for alternative constructive proofs of many theoretical results and thus lead to a much closer intertwining of computation and theory than ordinary Gröbner bases.

Pommaret bases are a particular form of involutive bases which in turn represent a special kind of Gröbner bases with additional combinatorial properties. Involutive bases combine ideas of the Janet-Riquier theory of partial differential equations with Gröbner bases. Zharkov and Blinkov [51] introduced Pommaret bases as involutive polynomial bases into commutative algebra. Later, Gerdt, Blinkov, Zharkov, and others developed a general theory of involutive bases [20. The terminology Pommaret bases is historically incorrect, as they appear already in the work of Janet (see e.g. [27, pp. 30-31]); however, the name has become standard by now. For a general survey on involutive bases with special emphasis on Pommaret bases see e.g. 44, 45, 46, (the last reference also contains some historical notes).

In the sequel, we will apply some of the above mentioned results to effective algebraic geometry. Bézout's theorem may be considered as a generalization of the fundamental theorem of algebra. Let $f_{1}, \ldots, f_{n-1} \in \mathcal{P}=\mathbb{k}\left[x_{1}, \ldots, x_{n}\right]$ be a sequence of homogeneous polynomials. If the system $f_{1}=\cdots=f_{n-1}=0$ has a finite number of projective solutions (i. e. the dimension as projective variety is zero), then the number of solutions, counted with multiplicities, is at most $\prod_{i=1}^{n-1} \operatorname{deg}\left(f_{i}\right)$, see [31, page 174]. One can consider higher-dimensional extensions of this result using the degree of an ideal. If the ideal $\mathcal{I}$ is generated by $k$ homogeneous polynomials of degrees $d_{1} \geq \cdots \geq d_{k}$, then $\operatorname{deg}(\mathcal{I}) \leq d_{1} \cdots d_{\mu}$ with $\mu=\min \{k, n\}$, see e.g. [8, Lem. 3], [24, Thm. 1] or [43, Lem. 2.95]. We will refer to this upper bound as Bézout's bound.

In this article, we are mainly concerned with two related topics. Firstly, we will express the degree of an ideal in terms of the degrees and classes of the elements of its Pommaret basis. As a by-product, we will provide a new proof for the rationality of the Hilbert series and an explicit formula for its numerator. Secondly, we will derive the new dimension depending Bézout bound $d_{1} \cdots d_{n-D}$ where $D=\operatorname{dim}(\mathcal{I})$ for the degree of $\mathcal{I}$. Masser and Wüstholz [36. Thm. II] proposed the upper bound $d_{1}^{n-D}$ (see also [3, Prop. 3.5] and [8]). However, our bound is sharper and our proof is more elementary. While the bound itself is independent of Pommaret bases, our proof relies crucially on special properties available only in quasi stable position, i. e. the generic position characterized by the existence of a finite Pommaret basis.

The article is organized as follows. In the next section, we review the basic definitions and notations which will be used throughout. Section 3 investigates the relationship between Pommaret bases and Hilbert series. In the last section, we derive our dimension depending Bézout bound and bound for the ideal membership problem, respectively. 


\section{Preliminaries}

We first introduce basic notations and preliminaries needed in the subsequent sections. Throughout $\mathcal{P}=\mathbb{k}\left[x_{1}, \ldots, x_{n}\right]$ will be the polynomial ring over an infinite field $\mathbb{k}$. We consider always homogeneous polynomials $f_{1}, \ldots, f_{k} \in \mathcal{P}$ and the ideal $\mathcal{I}=\left\langle f_{1}, \ldots, f_{k}\right\rangle$ generated by them. We assume that each $f_{i}$ is non-zero and denote its total degree by $d_{i}$. We sort the $f_{i}$ so that $d_{1} \geq$ $d_{2} \geq \cdots \geq d_{k}$ and we set $d=d_{1}$. Furthermore, the dimension of $\mathcal{I}$, denoted by $D=\operatorname{dim}(\mathcal{I})$, is the Krull dimension 11 of the corresponding factor ring $\mathcal{R}=\mathcal{P} / \mathcal{I}$. It is trivial that $k \geq n-D$. If $f \in \mathcal{P}$, the equivalence class of $f$ w.r.t. the congruence relation modulo $\mathcal{I}$ is an element of $\mathcal{R}$ and is denoted by $[f]$. Finally, we work throughout with the degree reverse lexicographic term order with $x_{n} \prec \cdots \prec x_{1}$.

The leading term of a polynomial $0 \neq f \in \mathcal{P}$, denoted by $\operatorname{LT}(f)$, is the greatest term appearing in $f$ and its coefficient is the leading coefficient, denoted by $\mathrm{LC}(f)$; the leading monomial is the product $\operatorname{LM}(f)=\mathrm{LC}(f) \operatorname{LT}(f)$. The leading ideal of $\mathcal{I}$ is the monomial ideal $\operatorname{LT}(\mathcal{I})=\langle\operatorname{LT}(f) \mid 0 \neq f \in \mathcal{I}\rangle$. A finite subset $\left\{g_{1}, \ldots, g_{m}\right\} \subset \mathcal{I}$ is called a Gröbner basis of $\mathcal{I}$ for $\prec$, if $\operatorname{LT}(\mathcal{I})=\langle\operatorname{LT}(G)\rangle=\langle\operatorname{LT}(g) \mid g \in G\rangle$. We refer to 4,12, for more details on Gröbner bases. We will denote by $\operatorname{deg}(\mathcal{I}, \prec)$ the maximal degree of the elements of the reduced Gröbner basis of $\mathcal{I}$ with respect to $\prec$ (see 33, 34, 21]).

For a positive integer $s$, we denote by $\mathcal{R}_{s}$ the set of elements of the factor ring $\mathcal{R}$ of degree $s$. Then the Hilbert function of $\mathcal{I}$ is defined by $\mathrm{HF}_{\mathcal{I}}(t)=$ $\operatorname{dim}_{\mathbb{k}}\left(\mathcal{R}_{t}\right)$ where $\operatorname{dim}_{\mathbb{k}}$ denotes the dimension as a $\mathbb{k}$-vector space. From a certain degree on, this function of $t$ is equal to a (unique) polynomial in $t$, called Hilbert polynomial, and denoted by $\mathrm{HP}_{\mathcal{I}}$. The Hilbert regularity of $\mathcal{I}$ is

$$
\operatorname{hilb}(\mathcal{I})=\min \left\{m \mid \forall t \geq m, \operatorname{HF}_{\mathcal{I}}(t)=\operatorname{HP}_{\mathcal{I}}(t)\right\}
$$

We have the identity $\operatorname{dim}(\mathcal{I})=\operatorname{deg}\left(\mathrm{HP}_{\mathcal{I}}\right)+1$, see [12, Thm. 12, page 464] and by Macaulay's theorem $\mathrm{HF}_{\mathcal{I}}=\mathrm{HF}_{\mathrm{LT}(\mathcal{I})}$.

The Hilbert series of $\mathcal{I}$ is the power series $\operatorname{HS}_{\mathcal{I}}(t)=\sum_{s=0}^{\infty} \operatorname{HF}_{\mathcal{I}}(s) t^{s}$. This series can be expressed as a quotient $\mathrm{HS}_{\mathcal{I}}(t)=N(t) /(1-t)^{D}$ with a polynomial $N \in \mathbb{Q}[t]$ satisfying $N(1) \neq 0$ (see [18, Thm. 7, page 130] or [50]). In the next section, we will provide a new proof of this fact using Pommaret bases.

Definition 2.1 ([22, page 52]) If $D>0$, then the degree of $\mathcal{I}$, denoted by $\operatorname{deg}(\mathcal{I}) 2$ is $(D-1)$ ! times the leading coefficient of the Hilbert polynomial of $\mathcal{I}$. If $D=0$, then $\operatorname{deg}(\mathcal{I})$ is defined to be the sum of the coefficients of $\operatorname{HS}_{\mathcal{I}}(t)$.

Remark 2.2 By [31, page 173], we have $\operatorname{deg}(\mathcal{I})=N(1)$ and in consequence since $\mathcal{I}$ and $\operatorname{LT}(\mathcal{I})$ share the same Hilbert function, $\operatorname{deg}(\mathcal{I})=\operatorname{deg}(\operatorname{LT}(\mathcal{I}))$. We

\footnotetext{
1 Note that the Krull dimension corresponds to the dimension as affine and not as projective variety, although we work exclusively with homogeneous ideals. We stick with the affine picture to facilitate comparison with other results which are also based on the dimension as affine variety.

2 Please note that despite the similarity in notation $\operatorname{deg}(\mathcal{I})$ and $\operatorname{deg}(\mathcal{I}, \prec)$ refer to very different objects!
} 
also need the following auxiliary results on the degree of an ideal from 8 . Let $Q$ be a $\mathfrak{p}$-primary ideal. We say that $Q$ has length $\ell$, if there exists a chain

$$
Q=Q_{1} \subset \cdots \subset Q_{\ell}=\mathfrak{p}
$$

of primary ideals $Q_{1}, \ldots, Q_{\ell}$, but no longer chain of this form. Let $r$ be the least positive integer $a$ such that $\mathfrak{p}^{a} \subset Q$. With these notations, we find that $r \leq \ell$ and $\operatorname{deg}(Q)=\ell \operatorname{deg}(\mathfrak{p})$, cf. [8, page 282]. Furthermore, the degree of $\mathcal{I}$ is equal to the sum of the degrees of its primary components of dimension $D$.

Let $\mathfrak{m}=\left\langle x_{1}, \ldots, x_{n}\right\rangle$ be the unique homogeneous maximal ideal of $\mathcal{P}$. The ideal $\mathcal{I}^{\text {sat }}=\mathcal{I}: \mathfrak{m}^{\infty}$ is called the saturation of $\mathcal{I}$. The satiety of $\mathcal{I}$, denoted by $\operatorname{sat}(\mathcal{I})$, is the smallest positive integer $m$ such that $\mathcal{I}_{\ell}=\mathcal{I}_{\ell}^{\text {sat }}$ for all $\ell \geq m$. It is always a finite number [1, Rem. 1.3]. As a trivial consequence, $\mathcal{I}$ and $\mathcal{I}^{\text {sat }}$ possess the same Hilbert polynomial and thus in particular the same degree. By [1, Lem. 1.6], $\mathcal{I}^{\text {sat }}=\mathcal{I}: y^{\infty}$ for a generic linear form $y$.

Definition 2.3 The ideal $\mathcal{I}$ is $m$-regular, if its $i$ th syzygy module can be generated by elements of degree at most $m+i$. The Castelnuovo-Mumford regularity $\operatorname{reg}(\mathcal{I})$ is the smallest $m$ such that $\mathcal{I}$ is $m$-regular.

For more details on the regularity, we refer to $[1,5$. The polynomials $f_{1}, \ldots, f_{k} \in \mathcal{P}$ form an $\mathcal{I}$-regular sequence for an ideal $\mathcal{I}$, if they generate a proper ideal in $\mathcal{P}$ and if $\left[f_{j}\right]$ is a non zero divisor in the $\operatorname{ring} \mathcal{P} /\left\langle\mathcal{I}, f_{1}, \ldots, f_{j-1}\right\rangle$ for $j=1, \ldots, k$. We simply speak of a regular sequence, if $\mathcal{I}=0$. The depth of $\mathcal{I}$, denoted by $\operatorname{depth}(\mathcal{I})$ is the maximal length of an $\mathcal{I}$-regular sequence.

Given a polynomial $f \in \mathcal{P}$ with $\operatorname{LT}(f)=x^{\alpha}$ where $\alpha=\left(\alpha_{1}, \ldots, \alpha_{n}\right)$, the class of $f$ is the integer $\operatorname{cls}(f)=\max \left\{i \mid \alpha_{i} \neq 0\right\}$. Then the multiplicative variables of $f$ are $\mathcal{X}_{P}(f)=\left\{x_{\operatorname{cls}(f)}, \ldots, x_{n}\right\}$ 3 The term $x^{\beta}$ is a Pommaret divisor of $x^{\alpha}$, written $\left.x^{\beta}\right|_{P} x^{\alpha}$, if $x^{\beta} \mid x^{\alpha}$ and $x^{\alpha-\beta} \in \mathbb{k}\left[\mathcal{X}_{P}\left(x^{\beta}\right)\right]$.

Definition 2.4 Let $\mathcal{H} \subset \mathcal{I}$ be a finite set such that no leading term of an element of $\mathcal{H}$ is a Pommaret divisor of the leading term of another element. Then $\mathcal{H}$ is a Pommaret basis of $\mathcal{I}$, if

$$
\bigoplus_{h \in \mathcal{H}} \mathbb{k}\left[\mathcal{X}_{P}(h)\right] \cdot \operatorname{LT}(h)=\operatorname{LT}(\mathcal{I}) .
$$

If an ideal $\mathcal{I}$ has a Pommaret basis $\mathcal{H}$, then $\operatorname{reg}(\mathcal{I})$ equals the maximal degree of an element of $\mathcal{H}$ and $\operatorname{depth}(\mathcal{I})$ is given by $n$ minus the maximal class of an element of $\mathcal{H}$. Furthermore $\mathcal{I}^{\text {sat }}=\mathcal{I}: x_{n}^{\infty}$ and the satiety is the maximal degree of an element of class $n$ in $\mathcal{H}$. We refer the reader to [44,45] and 46, Chap. 3-5] for a thorough introduction into the theory of Pommaret bases.

It follows immediately from the definition that any Pommaret basis is a (generally non-reduced) Gröbner basis. The main difference between Gröbner

\footnotetext{
3 We follow here the conventions of 20. In 45, a convention is used which corresponds to reverting the order of the variables $x_{1}, \ldots, x_{n}$. This implies e. g. that the class is defined as the minimum and not the maximum. Thus care must be taken when transferring results of 45] to the conventions used in this article.
} 
and Pommaret bases lies in the fact that any polynomial $f \in \mathcal{I}$ has a unique involutive standard representation, i.e. a standard representation where all coefficients depend only on the multiplicative variables of the respective generator, by the following direct sum decomposition as graded $\mathbb{k}$-linear spaces:

$$
\bigoplus_{h \in \mathcal{H}} \mathbb{k}\left[\mathcal{X}_{P}(h)\right] \cdot h=\mathcal{I}
$$

It furthermore allows to read off immediately the volume function of $\mathcal{I}$

$$
\operatorname{VF}_{\mathcal{I}}(t)=\operatorname{dim}_{\mathbb{k}}\left(\mathcal{I}_{t}\right)=\sum_{h \in \mathcal{H}}[\operatorname{deg}(h) \leq t]\left(\begin{array}{c}
t-\operatorname{deg}(h)+\left|\mathcal{X}_{P}(h)\right|-1 \\
t-\operatorname{deg}(h)
\end{array}\right)
$$

where [.] denotes the Kronecker-Iverson symbol which yields 1 , if the condition in the bracket is satisfied, and 0 otherwise. Obviously, the volume function is closely related to the Hilbert function: $\mathrm{HF}_{\mathcal{I}}=\mathrm{VF}_{\mathcal{P}}-\mathrm{VF}_{\mathcal{I}}$. Thus we obtain without any further computation the Hilbert function from a Pommaret basis. The same is true of the Hilbert polynomial: the volume polynomial $\mathrm{VP}_{\mathcal{I}}$ is given by the same expression as $\mathrm{VF}_{\mathcal{I}}$ with only the Kronecker-Iverson symbol omitted and then $\mathrm{HP}_{\mathcal{I}}=\mathrm{VP}_{\mathcal{P}}-\mathrm{VP}_{\mathcal{I}}$.

While this observation allows for an easy computation of both Hilbert function and polynomial for any concrete ideal possessing a Pommaret basis, it is not satisfying from a theoretical point of view. Via (1) we have only easy access to the volume function; the dependence of properties of the Hilbert function and related invariants like the degree on properties of the Pommaret basis is more difficult to assess. Therefore, we will exhibit this relationship in more detail in the next section 4

Unfortunately, Pommaret bases do not always exist. However, this is only a question of the used variables: since we assume that $\mathbb{k}$ is an infinite field, any ideal possesses a Pommaret basis after a generic linear change of variables 45. More precisely, we meet here the combinatorial notion of quasi-stability 5

Definition 2.5 A monomial ideal $\mathcal{J}$ is called quasi stable, if for any term $m \in \mathcal{J}$ and all integers $i, j, s$ with $1 \leq j<i \leq n$ and $s>0$, if $x_{i}^{s} \mid m$ there exists an integer $t \geq 0$ such that $x_{j}^{t} m / x_{i}^{s} \in \mathcal{J}$. The polynomial ideal $\mathcal{I}$ is in quasi stable position if $\operatorname{LT}(\mathcal{I})$ is quasi stable.

Proposition 2.6 (45]) A monomial ideal $\mathcal{J}$ has a Pommaret basis, if and only if it is quasi stable. A polynomial ideal $\mathcal{I}$ has a Pommaret basis, if and only if it is in quasi stable position.

\footnotetext{
4 In 45 46] also complementary decompositions, i.e. direct sum decompositions of the complement of $\operatorname{LT}(\mathcal{I})$ are discussed and it is shown that any Pommaret basis induces one. Then one can write down an explicit formula for $\mathrm{HF}_{\mathcal{I}}$ with a similar structure as (2). However, this only transforms the problem into understanding the precise relationship between the complementary decomposition and the Pommaret basis. While this is relatively simple with regard to, say, $\operatorname{dim}(\mathcal{I})$ and $\operatorname{depth}(\mathcal{I})$ (see the corresponding results in [45 46]), the situation becomes non-trivial for $\operatorname{deg}(\mathcal{I})$.

5 Quasi stable ideals are also known by many other names like weakly stable ideals, ideals of nested type or ideals of Borel type.
} 
Remark 2.7 It is trivial to see that all the objects studied in this work like the Hilbert function or the regularity remain invariant under linear changes of coordinates. Hence we may in the sequel always assume that we are in quasi stable position and thus that $\mathcal{I}$ has a Pommaret basis $\mathcal{H}$.

In the sequel, we will use the following notations: given an ideal $\mathcal{I}$ in quasi stable position, we write $\mathcal{H}=\left\{h_{1}, \ldots, h_{s}\right\}$ for its Pommaret basis. Furthermore, for each $i$ we set $m_{i}=\operatorname{LT}\left(h_{i}\right), c_{i}=\operatorname{deg}\left(m_{i}\right)$ and $n_{i}=n-\left|\mathcal{X}_{P}\left(m_{i}\right)\right|$, the number of non-multiplicative variables of $m_{i}$. By definition, $\mathcal{X}_{P}\left(h_{i}\right)=\mathcal{X}_{P}\left(m_{i}\right)$ and hence $\left\{m_{1}, \ldots, m_{s}\right\}$ forms a Pommaret basis for $\operatorname{LT}(\mathcal{I})$.

\section{Pommaret Bases and Hilbert Series}

We now study the relationship between the Pommaret basis $\mathcal{H}$ of a polynomial ideal $\mathcal{I}$ and certain invariants of $\mathcal{I}$ related to its Hilbert series. Our main results are new formulae expressing $\operatorname{deg}(\mathcal{I})$ and the coefficients of the numerator $N$ of the Hilbert series in terms of the degrees $c_{i}$ and the numbers $n_{i}$ of nonmultiplicative variables of the elements $h_{i}$ of $\mathcal{H}$. As a by-product, we provide new proofs of some classical results like the following one.

Theorem 3.1 The Hilbert series can be written as a rational function of the form $\operatorname{HS}_{\mathcal{I}}(t)=N(t) /(1-t)^{D}$ with a polynomial $N \in \mathbb{Z}[t]$ satisfying $N(1) \neq 0$.

Proof By Rem. 2.7, we may assume that $\mathcal{I}$ is in quasi stable position. It is easy to see that the Taylor coefficient of order $q$ of the function $1 /(1-t)^{n}$ and the number of terms of degree $q$ in $n$ variables coincide and thus the Hilbert series of the full polynomial ring is given by $1 /(1-t)^{n}$. It then follows from (2) and the subsequent discussion that

$$
\mathrm{HS}_{\mathcal{I}}(t)=\frac{1}{(1-t)^{n}}-\sum_{i=1}^{s} \frac{t^{\operatorname{deg}\left(m_{i}\right)}}{(1-t)^{\left|\mathcal{X}_{P}\left(m_{i}\right)\right|}}=\frac{1-\sum_{i=1}^{s}(1-t)^{n_{i}} t^{c_{i}}}{(1-t)^{n}}
$$

We number the generators $h_{i}$ such that $m_{1}, \ldots, m_{\ell} \in \mathbb{k}\left[x_{1}, \ldots, x_{n-D}\right]$ and $m_{\ell+1}, \ldots, m_{s} \notin \mathbb{k}\left[x_{1}, \ldots, x_{n-D}\right]$. Since $\mathcal{I}$ is in quasi stable position, the set $\mathcal{B}=\left\{m_{1}, \ldots, m_{\ell}\right\}$ contains pure powers of the variables $x_{1}, \ldots, x_{n-D}$. Hence, if we consider the contraction $\mathcal{J}=\mathrm{LT}(\mathcal{I}) \cap \mathcal{P}^{\prime}$ with $\mathcal{P}^{\prime}=\mathbb{k}\left[x_{1}, \ldots, x_{n-D}\right]$, then $\mathcal{B}$ is its Pommaret basis which trivially implies that $\mathcal{J}$ is zero-dimensional. It follows that the Hilbert series of $\mathcal{J}$ is a polynomial $P(t)$ with $P(1) \neq 0$ and the Hilbert series of the extension ideal $\mathcal{J}^{e}=\langle\mathcal{J}\rangle_{\mathcal{P}} \subset \mathcal{P}$ is given by $P(t) /(1-t)^{D}$.

On the other hand, the Pommaret basis $\mathcal{B}$ induces the direct sum decomposition $\mathcal{J}^{e}=\bigoplus_{i=1}^{\ell} \mathbb{k}\left[\mathcal{X}_{P}\left(m_{i}\right)\right] \cdot m_{i}$ and hence

$$
\frac{P(t)}{(1-t)^{D}}=\frac{1-\sum_{i=1}^{\ell}(1-t)^{n_{i}} t^{c_{i}}}{(1-t)^{n}}
$$


Consequently, $1-\sum_{i=1}^{\ell}(1-t)^{n_{i}} t^{c_{i}}=(1-t)^{n-D} P(t)$. Our assumptions imply that for $i=\ell+1, \ldots, s$ the term $m_{i}$ contains at least one of the variables $x_{n-D+1}, \ldots, x_{n}$ entailing that all the variables $x_{1}, \ldots, x_{n-D}$ are nonmultiplicative for it and thus $n_{i} \geq n-D$. It follows that the Hilbert series of $\mathcal{I}$ can be expressed in the form

$$
\frac{(1-t)^{n-D} P(t)-\sum_{i=\ell+1}^{s}(1-t)^{n_{i}} t^{c_{i}}}{(1-t)^{n}}=\frac{P(t)-\sum_{i=\ell+1}^{s}(1-t)^{n_{i}-n+D} t^{c_{i}}}{(1-t)^{D}} .
$$

Writing $N(t)$ for the numerator of the quotient on the right hand side, we thus obtain the rational form $\operatorname{HS}_{\mathcal{I}}(t)=N(t) /(1-t)^{D}$.

There only remains to show that $N(1) \neq 0$. For $n_{i}-n+D>0$ the polynomial $(1-t)^{n_{i}-n+D} t^{c_{i}}$ vanishes at $t=1$. Thus it suffices to consider only $P(t)$ (a polynomial with non-negative integer coefficients) and the polynomials $-(1-t)^{n_{i}-n+D} t^{c_{i}}$ for those indices $\ell+1 \leq i \leq s$ with $n_{i}-n+D=0$ (which at $t=1$ yields -1 ). The condition $n_{i}-n+D=0$ corresponds to a leading term of the form $m_{i}=x_{1}^{\alpha_{1}} \cdots x_{n-D}^{\alpha_{n-D}} x_{n-D+1}^{\alpha_{n-D+1}}$ with $\alpha_{n-D+1} \neq 0$. Since $\left\{m_{1}, \ldots, m_{s}\right\}$ forms a Pommaret basis, $x_{1}^{\alpha_{1}} \cdots x_{n-D}^{\alpha_{n-D}} \notin \mathcal{J}$ and therefore we can associate the unique non-zero class $\left[x_{1}^{\alpha_{1}} \cdots x_{n-D}^{\alpha_{n-D}}\right]$ in the quotient ring $\mathcal{P}^{\prime} / \mathcal{J}$ to each $m_{i}$ with $n_{i}-n+D=0$. Since $\operatorname{dim}(\mathcal{I})=D$, there is no pure power of $x_{n-D+1}$ contained in $\mathcal{I}$ and thus we cannot find any $m_{i}$ with $n_{i}-n+D=0$ which would correspond to the class [1]. Therefore, the number of leading terms $m_{i}$ with $n_{i}-n+D=0$ is strictly less than $\operatorname{dim}_{\mathbb{k}}\left(\mathcal{P}^{\prime} / \mathcal{J}\right)=P(1)$ and $N(1) \neq 0$.

Remark 3.2 The basic idea underlying the above proof, namely to use a direct sum decomposition for obtaining information about invariants like the Hilbert series is very old and goes back at least to Riquier [42] and Janet 28] (in the context of partial differential equations). In fact, Janet gave already an explicit formula for the Hilbert function in terms of what is nowadays called a Janet basis. Within algebra, it was mainly Stanley 48 who exploited much later the same idea. For this reason one often speaks of Stanley decompositions. However, the special decompositions induced by Pommaret bases appeared already considerably earlier in the work of Rees [41. These decompositions can also be used for the construction of resolutions, as Eliahou and Kervaire 17] showed first for the special case of stable ideals where they could obtain an explicit expression for the minimal resolution of the ideal. As a by-product, they obtained this way via the Betti numbers (3) for this special case. Later it was shown in 45. how their construction embeds into the theory of Pommaret bases and how it can consequently be generalised to quasi-stable ideals and (to some extent) to polynomial ideals in quasi-stable position. However, in these more general situations one no longer obtains the minimal resolution and hence only upper bounds for the Betti numbers. But as (3) is a simple consequence of the direct sum decomposition induced by the Pommaret basis independent of any explicit expression for the Betti numbers, it remains valid.

Based on the proof above, we can derive an upper bound for the degree of the numerator $N$ of the Hilbert series. Furthermore, we provide an explicit formula for the coefficients of $N$ in terms of the Pommaret basis $\mathcal{H}$. 
Proposition 3.3 Let $\mathrm{HS}_{\mathcal{I}}(t)=N(t) /(1-t)^{D}$ where $N(t)=a_{0}+a_{1} t+\cdots+a_{\ell} t^{\ell}$ with $a_{\ell} \neq 0$. If $\mathcal{H}$ is the Pommaret basis of $\mathcal{I}$, then $\operatorname{deg}(N)=\ell$ satisfies

$$
\ell \leq \max \left\{\operatorname{deg}(h)-\left|\mathcal{X}_{P}(h)\right|+D \mid h \in \mathcal{H}\right\}
$$

and the coefficients $a_{i}$ are given by

$$
\begin{aligned}
a_{i}= & \left(\begin{array}{c}
n-D+i-1 \\
n-D-1
\end{array}\right)-\sum_{\substack{h \in \mathcal{H}, \operatorname{deg}(h) \leq i,\left|\mathcal{X}_{P}(h)\right| \leq D}}(-1)^{i-\operatorname{deg}(h)}\left(\begin{array}{c}
D-\left|\mathcal{X}_{P}(h)\right| \\
i-\operatorname{deg}(h)
\end{array}\right) \\
& -\sum_{\substack{h \in \mathcal{H}, \operatorname{deg}(h) \leq i,\left|\mathcal{X}_{P}(h)\right|>D}}\left(\begin{array}{c}
\left|\mathcal{X}_{P}(h)\right|-D+i-\operatorname{deg}(h)-1 \\
\left|\mathcal{X}_{P}(h)\right|-D-1
\end{array}\right) .
\end{aligned}
$$

Proof By the proof of Thm. 3.1, $N(t)(1-t)^{n-D}=1-\sum_{j=1}^{s}(1-t)^{n_{j}} t^{c_{j}}$ and thus $\operatorname{deg}(N(t)) \leq \max \left\{n_{j}+c_{j} \mid j=1, \ldots, s\right\}-n+D$ which immediately implies the bound for $\ell$.

By the equality above, $N(t)=(1-t)^{D-n}-\sum_{j=1}^{s}(1-t)^{n_{j}-n+D} t^{c_{j}}$. Thus we have expressed $N$ as a sum of rational functions and now study the respective Taylor series. The coefficient of $t^{i}$ in the series expansion of $(1-t)^{D-n}$ is equal to the number of terms of degree $i$ in $n-D$ variables and hence to $\left(\begin{array}{c}n-D+i-1 \\ n-D-1\end{array}\right)$, the first term in (4). Now we must find the coefficient of $t^{i}$ in $(1-t)^{n_{j}-n+D} t^{c_{j}}$. Obviously, it vanishes for $c_{j}>i$. For $c_{j} \leq i$ two cases arise. If $n_{j}-n+D \geq 0$ (or equivalently $\left|\mathcal{X}_{P}(h)\right| \leq D$ ), then, by binomial expansion, the coefficient of $t^{i}$ is $(-1)^{i-c_{j}}\left(\begin{array}{c}n_{j}-n+D \\ i-c_{j}\end{array}\right)$ which yields the second summand in (4). Otherwise, the coefficient of $t^{i}$ is equal to the number of terms of degree $i-c_{j}$ in $n-n_{j}-D$ variables and thus to $\left(\begin{array}{c}n-n_{j}-D+i-c_{j}-1 \\ n-n_{j}-D-1\end{array}\right)$ leading to the last summand in (4).

The above result leads to two simple corollaries relating the Hilbert regularity with the parameters of the Pommaret basis and other invariants.

Corollary 3.4 If $\mathcal{H}$ is the Pommaret basis of $\mathcal{I}$, then

$$
\operatorname{hilb}(\mathcal{I}) \leq \max \left\{0, \operatorname{deg}(h)-\left|\mathcal{X}_{P}(h)\right|+1 \mid h \in \mathcal{H}\right\} .
$$

Proof Write $\operatorname{HS}_{\mathcal{I}}(t)=N(t) /(1-t)^{D}$ with $N(1) \neq 0$. It is well-known that $\operatorname{hilb}(\mathcal{I})=\max \{0, \operatorname{deg}(N(t))-D+1\}$, see e. g. 9, Prop. 4.1.12]. Now Prop. 3.3 immediately entails the assertion.

Corollary 3.5 Assume that the Pommaret basis $\mathcal{H}$ of $\mathcal{I}$ contains a generator $h \in \mathcal{H}$ having simultaneously the maximal degree and the maximal class among all elements of $\mathcal{H}$. Then

(1) $\operatorname{hilb}(\mathcal{I})=\max \left\{0, \operatorname{deg}(h)-\left|\mathcal{X}_{P}(h)\right|+1\right\}$,

(2) $\operatorname{deg}(N(t))=\operatorname{deg}(h)-\left|\mathcal{X}_{P}(h)\right|+D$,

(3) $\operatorname{hilb}(\mathcal{I})+\operatorname{depth}(\mathcal{I})=\max \{\operatorname{depth}(\mathcal{I}), \operatorname{reg}(\mathcal{I})\}$. 
Proof We may assume that $h=h_{s}$. Since $n_{s}+c_{s} \geq n_{i}+c_{i}$ for all $i$, two first equalities follow from the proofs of Prop. 3.3 and Cor. 3.4. respectively. By 45. Prop. 3.19] we have $\operatorname{depth}(\mathcal{I})=\left|\mathcal{X}_{P}\left(h_{s}\right)\right|-1$ and $\operatorname{reg}(\mathcal{I})=\operatorname{deg}\left(h_{s}\right)$ by 45 , Thm. 9.2] which entails the last assertion.

Remark 3.6 Mora [40, Thm. 38.1.1] claimed the equality $\operatorname{reg}(\mathcal{I})=\operatorname{hilb}(\mathcal{I})+$ $\operatorname{depth}(\mathcal{I})$ for arbitrary ideals. However, it does not even necessarily hold for ideals in generic position 6 . As a concrete counterexample, we consider the monomial ideal $\mathcal{I}=\left\langle x_{1} x_{3}, x_{1} x_{2}, x_{1}^{2}, x_{2}^{3}\right\rangle \subset \mathbb{k}\left[x_{1}, x_{2}, x_{3}\right]$ which is a generic initial ideal. Its generating set is already its Pommaret basis and therefore $\operatorname{reg}(\mathcal{I})=3$. The only term of maximal class is not of maximal degree and thus Cor. 3.5 cannot be invoked. , By [45, Prop. 3.19], $\operatorname{depth}(\mathcal{I})=0$. On the other hand, $\operatorname{HS}_{\mathcal{I}}(t)=-(2 t+1) /(-1+t)$ and therefore $\operatorname{hilb}(\mathcal{I})=1$. Thus $\operatorname{reg}(\mathcal{I})>\operatorname{hilb}(\mathcal{I})+\operatorname{depth}(\mathcal{I})$. Finally, we note that in general the inequality $\operatorname{reg}(\mathcal{I}) \geq \operatorname{hilb}(\mathcal{I})+\operatorname{depth}(\mathcal{I})$ does not hold. For example, consider the above ideal in the ring $\mathbb{k}\left[x_{1}, \ldots, x_{7}\right]$. Then, $\operatorname{reg}(\mathcal{I})=3, \operatorname{hilb}(\mathcal{I})=0$ and $\operatorname{depth}(\mathcal{I})=4$.

Finally, we provide an explicit expression for the degree of an ideal in terms of its Pommaret basis. Below, we denote by $f^{(i)}$ the $i$ th derivative of the function $f$.

Theorem 3.7 Let $\mathcal{H}$ be the Pommaret basis of $\mathcal{I}$. Then

$$
\operatorname{deg}(\mathcal{I})=\sum_{\substack{h \in \mathcal{H}, D \leq\left|\mathcal{X}_{P}(h)\right| \leq D+\operatorname{deg}(h)}}(-1)^{\left|\mathcal{X}_{P}(h)\right|-D+1}\left(\begin{array}{c}
\operatorname{deg}(h) \\
\left|\mathcal{X}_{P}(h)\right|-D
\end{array}\right) .
$$

Proof We claim that

$$
\operatorname{deg}(\mathcal{I})=N(1)=\left.\frac{(-1)^{n-D}}{(n-D) !}\left(N(t)(1-t)^{n-D}\right)^{(n-D)}\right|_{t=1} .
$$

Indeed, by the general Leibniz rule we have

$$
\left(N(t)(1-t)^{n-D}\right)^{(n-D)}=\sum_{k_{1}+k_{2}=n-D} \frac{(n-D) !}{k_{1} ! k_{2} !} N(t)^{\left(k_{1}\right)}\left((1-t)^{n-D}\right)^{\left(k_{2}\right)}
$$

On the right hand side, all summands vanish at $t=1$ except for $k_{1}=0$ and $k_{2}=n-D$ which proves the claim.

By the proof of Thm. 3.1 $N(t)(1-t)^{n-D}=1-\sum_{i=1}^{s}(1-t)^{n_{i}} t^{c_{i}}$. Thus there only remains to determine the derivatives of the right hand side. Applying again the general Leibniz rule, we obtain

$$
\left((1-t)^{n_{i}} t^{c_{i}}\right)^{(n-D)}=\sum_{k_{1}+k_{2}=n-D} \frac{(n-D) !}{k_{1} ! k_{2} !}\left((1-t)^{n_{i}}\right)^{\left(k_{1}\right)}\left(t^{c_{i}}\right)^{\left(k_{2}\right)} .
$$

6 By generic position, we mean after a linear change of variables from a Zariski open set, see [1] for more details. 
At $t=1$, all summands disappear except for $k_{1}=n_{i} \leq n-D$ and $k_{2} \leq c_{i}$. Such indices appear whenever $n_{i}+c_{i} \geq n-D$ and $n_{i} \leq n-D$. By simple manipulations, one now obtains

$$
\left.\left((1-t)^{n_{i}} t^{c_{i}}\right)^{(n-D)}\right|_{t=1}=(-1)^{n_{i}}(n-D) !\left(\begin{array}{c}
c_{i} \\
n-D-n_{i}
\end{array}\right)
$$

which immediately yields the assertion.

\section{Dimension Depending Upper Bounds}

We now exploit some of the results obtained in the last section to derive a dimension depending upper bound for the degree of a homogeneous ideal. Furthermore, as related subjects, we provide new dimension depending upper bounds in the effective Nullstellensatz, in elimination theory and for the ideal membership problem. Let us quickly recall the used notations. $\mathcal{I} \subset \mathcal{P}$ is an ideal generated by the homogeneous polynomials $f_{1}, \ldots, f_{k}$. If $\mathcal{I}$ is in quasi stable position, then we denote its Pommaret basis by $\mathcal{H}$. We write $d_{i}=\operatorname{deg}\left(f_{i}\right)$ and assume that $d_{1} \geq \cdots \geq d_{k}$. If an index $i>k$ appears, then we set $d_{i}=1$. Then the classical Bézout bound (see e.g. [24, Thm. 1], [8, Lem. 3] or [43, Lem. 2.95]) asserts that $\operatorname{deg}(\mathcal{I}) \leq d_{1} \cdots d_{\mu}$ with $\mu=\min \{k, n\}$. We will now improve this bound using the dimension $7=\operatorname{dim} \mathcal{I}$.

Lemma 4.1 If the ideal $\mathcal{I}$ is in quasi stable position and $\mathcal{H}$ its Pommaret basis, then for each $i \leq n$ the set $\left.\mathcal{H}\right|_{x_{i}=\cdots=x_{n}=0}$ is the Pommaret basis of the ideal $\left.\mathcal{I}\right|_{x_{i}=\ldots=x_{n}=0} \subseteq \mathbb{k}\left[x_{1}, \ldots, x_{i-1}\right]$.

Proof Obviously, $\left.\left.\mathcal{H}\right|_{x_{n}=0} \subset \mathcal{I}\right|_{x_{n}=0}$ and no leading term of an element of $\left.\mathcal{H}\right|_{x_{n}=0}$ is a Pommaret divisor of the leading term of another element. It is wellknown that the reverse lexicographic term order implies that, if $x_{n}$ divides the leading term of a polynomial, then it divides every term in the polynomial. It follows immediately that $\left.\mathcal{I}\right|_{x_{n}=0}=\left.\bigoplus_{h \in \mathcal{H}} \mathbb{k}\left[\mathcal{X}_{P}\left(\left.h\right|_{x_{n}=0}\right)\right] \cdot h\right|_{x_{n}=0}$ and $\left.\mathcal{H}\right|_{x_{n}=0}$ is thus the Pommaret basis of $\left.\mathcal{I}\right|_{x_{n}=0}$. For $i<n$ the claim is established using a simple induction.

Proposition 4.2 Suppose that the ideal $\mathcal{I}$ is in quasi stable position and of dimension $D>0$. Then $\operatorname{deg}(\mathcal{I})=\operatorname{deg}\left(\left.\mathcal{I}\right|_{x_{n-D+2}=\cdots=x_{n}=0}\right)$.

Proof Let $\mathcal{H}$ be the Pommaret basis of $\mathcal{I}$. It follows from Thm. 3.7 that generators with class greater than $n-D+1$ are not considered in the there provided formula for $\operatorname{deg}(\mathcal{I})$. Together with Lem. 4.1, this observation implies that the degrees of $\mathcal{I}$ and of $\left.\mathcal{I}\right|_{x_{n-D+2}=\cdots=x_{n}=0}$ are identical.

7 Although we are dealing with a homogeneous ideal, we will always work with the dimension as affine variety. 
Example 4.3 Let $\mathcal{I}=\left\langle x_{2} x_{3}, x_{1}^{2}, x_{1} x_{2} x_{4}, x_{2}^{3}, x_{1} x_{3}^{2} x_{4}, x_{1} x_{3}^{3}, x_{2}^{2} x_{4}^{2} x_{5}, x_{4}^{3} x_{2}^{2}\right\rangle$ be an ideal in $\mathcal{P}=\mathbb{k}\left[x_{1}, \ldots, x_{5}\right]$. One can easily show that $\mathcal{I}$ is quasi stable and that $\operatorname{dim}(\mathcal{I})=3$. By Prop. 4.2, $\operatorname{deg}(\mathcal{I})=\operatorname{deg}\left(\left.\mathcal{I}\right|_{x_{4}=x_{5}=0}\right)=1$, and this makes the computation of the degree of $\mathcal{I}$ less expensive. We note that generally Prop. 4.2 becomes false, if we set further variables to zero. In our concrete example, one easily checks that the Hilbert series of $\left.\mathcal{I}\right|_{x_{3}=x_{4}=x_{5}=0}$ is $t^{3}+2 t^{2}+2 t+1$ and therefore $\operatorname{deg}\left(\left.\mathcal{I}\right|_{x_{3}=x_{4}=x_{5}=0}\right)=6 \neq \operatorname{deg}(\mathcal{I})$.

Corollary 4.4 If $D>0$ then $\operatorname{deg}(\mathcal{I}) \leq d_{1} \cdots d_{\mu}$ where $\mu=\min \{k, n-D+1\}$.

Proof By Rem. 2.7, we may assume that $\mathcal{I}$ is in quasi stable position. By Prop. 4.2, the degrees of $\mathcal{I}$ and of $\left.\mathcal{I}\right|_{x_{n-D+2}=\cdots=x_{n}=0}$ are identical with the latter ideal lying in the ring $\mathbb{k}\left[x_{1}, \ldots, x_{n-D+1}\right]$. Now the assertion follows by Bézout's bound.

Theorem 4.5 (Dimension depending Bézout bound) If the ideal $\mathcal{I}$ has dimension $D$, then $\operatorname{deg}(\mathcal{I}) \leq d_{1} \cdots d_{n-D}$.

Proof For $D=0$, this is just the classical Bézout bound. For $D>0$, we may assume that $\mathcal{I}$ is in quasi stable position by Rem. 2.7. By Prop. 4.2. $\operatorname{deg}(\mathcal{I})=\operatorname{deg}(\mathcal{J})$ where $\mathcal{J}=\left.\mathcal{I}\right|_{x_{n-D+2}=\cdots=x_{n}=0} \subseteq \mathbb{k}\left[x_{1}, \ldots, x_{n-D+1}\right]$ and it suffices to prove the desired upper bound for the latter ideal. Since $\mathcal{I}$ is in quasi stable position and $D=\operatorname{dim}(\mathcal{I})$, a pure power of each variable $x_{1}, \ldots, x_{n-D}$ appears in $\operatorname{LT}(\mathcal{I})$ and no pure power of $x_{n-D+1}$ belongs to $\operatorname{LT}(\mathcal{I})$ (this follows e. g. from [45, Prop. 3.15]). Therefore, $\operatorname{dim}(\mathcal{J})=1$.

$\mathcal{J}$ is a homogeneous ideal generated by the polynomials $\left.f_{i}\right|_{x_{n-D+2}=\cdots=x_{n}=0}$ with $i=1, \ldots, k$. Since $\mathcal{I}$ is in quasi stable position, $\mathcal{J}$ is in quasi stable position, too, (Lem. 4.1) and therefore $\mathcal{J}^{\text {sat }}=\mathcal{J}: x_{n-D+1}^{\infty}$ (see e.g. 45, Prop. 10.1]) . This implies that the degree of $\mathcal{J}^{\text {sat }}$ equals the number of projective solutions (with multiplicity) of the system associated to $\mathcal{J}^{\text {sat }}$ (see e. g. [33. Thm. 3.2]). Since the ideal $\mathcal{J}^{\text {sat }}$ is saturated, this number equals the number of affine solutions (with multiplicity) of the system associated to the ideal $\mathcal{J}$ with $x_{n-D+1}=1$. Obviously, the ideal $\left.\mathcal{J}\right|_{x_{n-D+1}=1}$ is zero-dimensional and generated by the polynomials $\left.f_{i}\right|_{x_{n-D+1}=1, x_{n-D+2}=\cdots=x_{n}=0}$ with $i=1, \ldots, k$ (see [45, Prop. 10.1]). By Bézout's theorem, the number of solutions is thus bounded by $d_{1} \cdots d_{n-D}$ and hence $\operatorname{deg}\left(\mathcal{J}^{\text {sat }}\right) \leq d_{1} \cdots d_{n-D}$. Our assertion now follows from the fact that $\operatorname{deg}(\mathcal{J})=\operatorname{deg}\left(\mathcal{J}^{\text {sat }}\right)$.

Remark 4.6 An alternative proof of Thm. 4.5 goes as follows. Suppose that the ideal $\mathcal{I}$ is in quasi stable position. As we observed, for bounding $\operatorname{deg}(\mathcal{I})$, we could only add the variables $x_{n-D+2}, \ldots, x_{n}$ into $\mathcal{I}$ and the main obstacle in estimating $\operatorname{deg}(\mathcal{I})$ was the addition of $x_{n-D+1}$ into $\left.\mathcal{I}\right|_{x_{n-D+2}=\cdots=x_{n}=0}$. Using the notations in the proof above, we have $\operatorname{deg}(\mathcal{I})=\operatorname{deg}(\mathcal{J})=\operatorname{deg}\left(\mathcal{J}^{\text {sat }}\right)$ and $\mathcal{J} \subset \mathbb{k}\left[x_{1}, \ldots, x_{n-D+1}\right]$ is a one-dimensional ideal. If $\mathcal{H}^{\prime}=\left.\mathcal{H}\right|_{x_{n-D+2}=\cdots=x_{n}=0}$ is the Pommaret basis of $\mathcal{J}$, then we set $\mathcal{H}_{1}^{\prime}=\left\{h \in \mathcal{H}^{\prime} \mid \operatorname{cls}(h)=n-D+1\right\}$ and $\overline{\mathcal{H}}_{1}^{\prime}=\left\{h / x_{n-D+1}^{\operatorname{deg}_{x_{n-D+1}}(h)} \mid h \in \mathcal{H}_{1}^{\prime}\right\}$ where $\operatorname{deg}_{x_{n-D+1}}(h)$ denotes the degree of $h$ in the variable $x_{n-D+1}$. [45, Prop. 10.1] asserts that $\mathcal{H}^{\prime} \backslash \mathcal{H}_{1}^{\prime} \cup \overline{\mathcal{H}}_{1}$ is a 
(non reduced) Pommaret basis (and thus a Gröbner basis) of $\mathcal{J}^{\text {sat }}$. It follows that $x_{n-D+1}$ does not appear in $\operatorname{LT}\left(\mathcal{J}^{\text {sat }}\right)$ and therefore $\operatorname{deg}(\mathcal{J})$ is equal to the degree of $\left.\mathcal{J}^{\text {sat }}\right|_{x_{n-D+1}=0} \subset \mathbb{k}\left[x_{1}, \ldots, x_{n-D}\right]$ which is a zero-dimensional ideal. On the other hand, we know that the ideal $\mathcal{K}$ generated by the polynomials $\left.f_{i}\right|_{x_{n-D+1}=x_{n-D+2}=\cdots=x_{n}=0}$ with $i=1, \ldots, k$ is zero-dimensional and $\mathcal{K} \subset$ $\left.\mathcal{J}^{\text {sat }}\right|_{x_{n-D+1}=0}$. This yields that $\operatorname{deg}\left(\left.\mathcal{J}^{\text {sat }}\right|_{x_{n-D+1}=0}\right) \leq \operatorname{deg}(\mathcal{K})$. So the desired inequality follows from Bézout's theorem. For example, the generating set of the ideal $\mathcal{I}=\left\langle 5 x_{2}^{2}+4 x_{2} x_{3}+x_{3}^{2}, 2 x_{1} x_{2}+x_{1} x_{3}+2 x_{2}^{2}+x_{2} x_{3}, x_{1}^{2}+x_{1} x_{2}, x_{1} x_{3}^{2}+x_{2} x_{3}^{2}\right\rangle$ in the polynomial ring $\mathbb{k}\left[x_{1}, x_{2}, x_{3}\right]$ is already a Pommaret basis and $\operatorname{dim}(\mathcal{I})=$ 1. By the above argument, the set $\mathcal{H}^{\prime}=\left\{5 x_{2}^{2}, 2 x_{1} x_{2}+2 x_{2}^{2}, x_{1}^{2}+x_{1} x_{2}, x_{1}+x_{2}\right\}$ is a Gröbner basis for $\mathcal{I}^{\text {sat }}$ and this observation entails $\operatorname{deg}(\mathcal{I})=2$.

Remark 4.7 In general, this upper bound improves the upper bound $d_{1}^{n-D}$ for $\operatorname{deg}(\mathcal{I})$ due to Masser and Wüstholz [36, Thm. II], cf. [3, Prop. 3.5]. We also note that Lazard [34, Prop. 1] presented (without any proof or reference) a similar upper bound.

Example 4.8 Consider the homogeneous ideal

$$
\begin{aligned}
\mathcal{I}= & \left\langle x_{5} x_{7}+x_{1} x_{8}, x_{6} x_{7}+x_{1} x_{9}, x_{6} x_{8}+x_{5} x_{9}, x_{5} x_{2}+x_{1} x_{3},\right. \\
& \left.x_{6} x_{2}+x_{1} x_{4}, x_{6} x_{3}+x_{5} x_{4}, x_{8} x_{2}+x_{7} x_{3}, x_{9} x_{2}+x_{7} x_{4}, x_{9} x_{3}+x_{8} x_{4}\right\rangle
\end{aligned}
$$

in the polynomial ring $\mathbb{k}\left[x_{1}, x_{2}, x_{3}, x_{4}, x_{5}, x_{6}, x_{7}, x_{8}, x_{9}\right]$ appearing in the work of Eisenbud and Sturmfels [16. We note that $\operatorname{dim}(\mathcal{I})=3, \operatorname{deg}(\mathcal{I})=24$ and $\mathcal{I}$ is generated by 9 quadratic polynomials. The classical Bézout bound yields the estimate $\operatorname{deg}(\mathcal{I}) \leq 2^{9}=512$, while Thm. 4.5 says $\operatorname{deg}(\mathcal{I}) \leq 2^{6}=64$.

Example 4.9 The so-called Mora-Lazard-Masser-Philippon-Kollár example [7] shows that the degree bound of Thm. 4.5 is sharp. For any sequence of degrees $d_{1}, \ldots, d_{n-1}$, let $\mathcal{I}$ by the ideal generated by the set

$$
A=\left\{x_{1}^{d_{1}}-x_{2} x_{n}^{d_{1}-1}, x_{2}^{d_{2}}-x_{3} x_{n}^{d_{2}-1}, \ldots, x_{n-2}^{d_{n-2}}-x_{n-1} x_{n}^{d_{n-2}-1}, x_{n-1}^{d_{n-1}}\right\} .
$$

The first Buchberger criterion (see e. g. [12]) shows easily that $A$ is the reduced Gröbner basis of $\mathcal{I}$. Therefore, $\operatorname{LT}(\mathcal{I})=\left\langle x_{1}^{d_{1}}, \ldots, x_{n-2}^{d_{n-2}}, x_{n-1}^{d_{n-1}}\right\rangle$ entailing that $\operatorname{HS}_{\mathcal{I}}(t)=\left(1-t^{d_{1}}\right) \cdots\left(1-t^{d_{n-1}}\right) /(1-t)^{n}$ and thus $\operatorname{deg}(\mathcal{I})=d_{1} \cdots d_{n-1}$.

We finally discuss some new dimension depending upper bounds for the effective Nullstellensatz, for elimination theory and for the ideal membership problem. We first briefly review some known results related to the effective Nullstellensatz that we will use in the rest of this section. For a sequence $d_{1} \geq \cdots \geq d_{k}$ of positive integers, let

$$
\mathrm{N}\left(d_{1}, \ldots, d_{k} ; n\right)= \begin{cases}\prod_{i=1}^{k} d_{i} & \text { if } n \geq k \geq 1 \\ d_{k} \prod_{i=1}^{n-1} d_{i} & \text { if } k>n>1 \\ d_{k} & \text { if } n=1\end{cases}
$$


In an effective Nullstellensatz, one considers statements as follows. If a homogeneous polynomial $f$ belongs to the radical of the ideal generated by the polynomials $f_{i}$, then there exists a positive integer $e$ and polynomials $g_{1}, \ldots, g_{k} \in \mathcal{P}$ such that $f^{e}=g_{1} f_{1}+\cdots+g_{k} f_{k}$ with $e \leq \mathrm{N}\left(d_{1}, \ldots, d_{k} ; n\right)$ and $\operatorname{deg}\left(g_{i} f_{i}\right) \leq$ $\operatorname{deg}(f) \mathrm{N}\left(d_{1}, \ldots, d_{k} ; n\right)$. This result also holds for non-homogeneous polynomials, if we replace the factor $\operatorname{deg}(f)$ by $1+\operatorname{deg}(f)$. The smallest integer $e$ such that for every polynomial $f \in \sqrt{\mathcal{I}}$ we have $f^{e} \in \mathcal{I}$ is called the Noether exponent of $\mathcal{I}$ and it is denoted by $\mathrm{e}(\mathcal{I})$.

Let $X$ be a variety of dimension $n$ and $\operatorname{deg}(X)$ its degree. Let $f_{1}, \ldots, f_{k} \in$ $\mathbb{k}[X]$ be a sequence of polynomials so that $d_{i}=\operatorname{deg}\left(f_{i}\right)$ with $d_{1} \geq \cdots \geq d_{k}$. Then, the above assertion holds true for the $f_{i}$ 's if in the above upper bound we replace $\mathrm{N}\left(d_{1}, \ldots, d_{k} ; n\right)$ by $\operatorname{deg}(X) \mathrm{N}\left(d_{1}, \ldots, d_{k} ; n\right)$. Thus, Thm. 4.5 could be useful for finding an upper bound for $\operatorname{deg}(X)$, if it is unknown. For further details on this topic, we refer to e.g. $32,49,29$.

We are now concerned with obtaining an upper bound for the Nother exponent $\mathrm{e}(\mathcal{I})$. For unmixed 8 ideals, it has been shown that $\mathrm{e}(\mathcal{I}) \leq d_{1} \cdots d_{n-D}$ cf. [8, Lem. 4]. However, this inequality does not hold in general.

Example 4.10 Consider the idea $9 \mathcal{I}=\left\langle x^{4}+x^{3} y, x^{3}+y^{3}\right\rangle \subset \mathbb{k}[x, y]$. We have $\mathcal{I}=\langle x+y\rangle \cap\left\langle x^{3}, y^{3}\right\rangle, \operatorname{dim}(\mathcal{I})=1$ and $d_{1}=4$. On the other hand, we note that $x+y \in \sqrt{I}$ however $(x+y)^{4} \notin \mathcal{I}$ and $(x+y)^{5} \in \mathcal{I}$. Therefore, $e \geq 5 \not \leq d_{1}=4$.

In characteristic zero, we now provide a refinement of this bound for all one-dimensional ideals using the next theorem due to Lazard [34, Thm. 2].

Theorem 4.11 Let the ideal $\mathcal{I}$ be in generic position and assume that $D \leq 1$. Then $\operatorname{deg}(\mathcal{I}, \prec) \leq d_{1}+\cdots+d_{r}-r+1$ where $r=n-\operatorname{depth}(\mathcal{I})$.

Proposition 4.12 Let $\mathcal{I}$ be an ideal of dimension $D=1$ over a field of characteristic zero. Then $\mathrm{e}(\mathcal{I}) \leq \max \left\{d_{1} \cdots d_{n-1}, d_{1}+\cdots+d_{r}-r+1\right\}$ where $r=n-\operatorname{depth}(\mathcal{I})$.

Proof W.l.o.g. we may assume that $\mathcal{I}$ is in generic position. Since $D=1$ and $\mathcal{I}$ is homogeneous, a primary decomposition $\mathcal{I}=Q_{1} \cap \cdots \cap Q_{s} \cap Q$ exists where each $Q_{i}$ is a $\mathfrak{p}_{i}$-primary component of $\mathcal{I}$ of dimension 1 and $Q$ is $\mathfrak{m}$-primary. Let $\ell_{i}$ be the length of $Q_{i}$. By Rem. 2.2, $\operatorname{deg}(\mathcal{I})=\sum_{i=1}^{s} \ell_{i} \operatorname{deg}\left(\mathfrak{p}_{i}\right)$. We note that $\sqrt{I}=\mathfrak{p}_{1} \cap \cdots \cap \mathfrak{p}_{s}$. For any polynomial $f \in \mathfrak{p}_{1} \cap \cdots \cap \mathfrak{p}_{s}$, one has $f^{\operatorname{deg}(\mathcal{I})} \in$ $Q_{1} \cap \cdots \cap Q_{s}$ (cf. Rem. 2.2). On the other hand, one easily sees that any $f \in \mathfrak{m}$ satisfies $f^{\operatorname{sat}(\mathcal{I})} \in Q$. Hence we find $\mathrm{e}(\mathcal{I}) \leq \max \{\operatorname{deg}(\mathcal{I})$, $\operatorname{sat}(\mathcal{I})\}$. Now the assertion follows from Thm. 4.11 and the fact that in generic (more precisely, in stable) position $\operatorname{sat}(\mathcal{I}) \leq \operatorname{reg}(\mathcal{I})=\operatorname{deg}(\mathcal{I}, \prec)$ (see e.g. [46, Thms. 5.5.7 and 5.5.15, Prop. 5.5.28]).

Example 4.13 We consider again the ideal of Example4.10, There $\operatorname{depth}(\mathcal{I})=$ 0 and by Prop. 4.12 we get $\mathrm{e}(\mathcal{I})=5 \leq \max \{4,4+3-2+1\}=6$.

\footnotetext{
8 An ideal is called unmixed if all its associated prime ideals have the same dimension.

9 This example has been provided by David Masser (private communication).
} 
Remark 4.14 The restriction to characteristic zero is required in the last step of the proof of Prop. 4.12. Only in characteristic zero we can always achieve stable position by a linear transformation. In positive characteristic $p>0$, the ideal $\mathcal{I}=\left\langle x_{1}^{p}, x_{2}^{p}\right\rangle \subset \mathbb{k}\left[x_{1}, x_{2}\right]$ is not in stable position and invariant under any linear transformation. For such ideals we only have the inequality $\operatorname{reg}(\mathcal{I}) \geq \operatorname{deg}(\mathcal{I}, \prec)$ and cannot conclude any relationship between sat $(\mathcal{I})$ and $\operatorname{deg}(\mathcal{I}, \prec)$. For the above ideal $\operatorname{sat}(\mathcal{I})=\operatorname{reg}(\mathcal{I})=2 p-1>\operatorname{deg}(\mathcal{I}, \prec)=p$. We get the same values for these invariants, if we consider $\mathcal{I}$ as an ideal in $\mathbb{k}\left[x_{1}, x_{2}, x_{3}\right]$ so that it is one-dimensional. Prop. 4.12 remains correct for this example, as the bound comes now from the first term which is $p^{2}$.

In the remainder of this section, unless explicitly stated otherwise, we skip the assumption that we are dealing with homogeneous polynomials and ideals, but keep otherwise our notations. We present first a dimension depending bound for the representation problem related to Nœther normalization [13. (see also [30,38]). Recall that an ideal $\mathcal{I} \subset \mathcal{P}$ is in Nother position, if the ring extension $K\left[x_{n-D+1}, \ldots, x_{n}\right] \hookrightarrow \mathcal{P} / \mathcal{I}$ is integral [15]. In this case, for each index $1 \leq i \leq n-D$, the intersection $\mathcal{I} \cap \mathbb{k}\left[x_{i}, x_{n-D+1}, \ldots, x_{n}\right]$ is non-empty and, by [13, Prop. 1.7], contains a witness polynomial $h_{i}$ which is monic in $x_{i}$ and can be represented in the form $h_{i}=g_{1} f_{1}+\cdots+g_{k} f_{k}$ with coefficients $g_{1}, \ldots, g_{k} \in \mathcal{P}$ such that $\operatorname{deg}\left(g_{j} f_{j}\right) \leq d^{n}\left(d^{n}+1\right)$ for $d=d_{1}$. In this estimate, the first factor $d^{n}$ represents an upper bound for $\mathrm{N}\left(d_{1}, \ldots, d_{k} ; n\right)$. Mayr and Ritscher [38] proved the following improvement.

Proposition 4.15 ([38, Thm. 10]) In the above described situation, the coefficients $g_{j}$ can be chosen such $\operatorname{deg}\left(g_{j} f_{j}\right) \leq\left(d_{1} \cdots d_{n-D}\right)^{2}$.

We will now improve this bound to $3 d_{1} \cdots d_{n-D}$ and study its application in the membership problem. We denote by ${ }^{h} \mathcal{P}$ the ring $\mathbb{k}\left[x_{1}, \ldots, x_{n+1}\right]$ where $x_{n+1}$ is a new variable. For any polynomial $f \in \mathcal{P}$, we consider its homogenization ${ }^{h} f=x_{n+1}^{\operatorname{deg}(f)} f\left(x_{1} / x_{n+1}, \ldots, x_{n} / x_{n+1}\right) \in{ }^{h} \mathcal{P}$. For an ideal $\mathcal{I} \subset \mathcal{P}$, its homogenization is defined as ${ }^{h} \mathcal{I}=\left\langle{ }^{h} f \mid f \in \mathcal{I}\right\rangle \subset{ }^{h} \mathcal{P}$. We need the following result due to Sombra [49, Lem. 3.15] which allows us to extract a regular sequence which remains regular after homogenization. We denote by $\mathcal{I}_{i}$, for $i=1, \ldots, k$, the ideal generated by $f_{1}, \ldots, f_{i}$ with the convention $\mathcal{I}_{0}=\langle 0\rangle$.

Lemma 4.16 ( $\sqrt{49}$, Lem. 3.15]) Let $f_{1}, \ldots, f_{k}$ be a regular sequence. Then polynomials $p_{1}, \ldots, p_{k}$ and $q_{1}, \ldots, q_{k}$ exist in $\mathcal{P}$ such that for any $i$

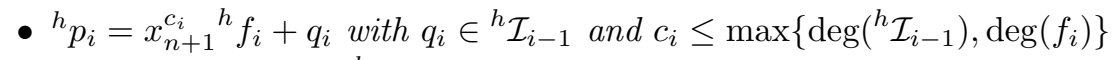

- $\operatorname{deg}\left(p_{i}\right) \leq \max \left\{\operatorname{deg}\left({ }^{h} \mathcal{I}_{i-1}\right), \operatorname{deg}\left(f_{i}\right)\right\}$

- ${ }^{h} p_{1}, \ldots,{ }^{h} p_{k}$ forms a regular sequence in ${ }^{h} \mathcal{P}$.

We remark that Sombra [49] assumed the conditions $d_{2} \geq \cdots \geq d_{k} \geq d_{1}$ which may make our next bounds sharper, however, using the fact that any permutation of a regular sequence is a regular sequence and for simplicity we continue with our restrictions on the degrees. We also use the following two results related to regular sequences. The next proposition can be found 
e. g. in [43, Lem. 2.81] or [38, Lem. 9]. However, we prove it for the sake of completeness. In the proof, we apply the well-known fact that the set of all zero-divisors for an ideal in $\mathcal{P}$ is the union of all its minimal prime ideals.

Proposition 4.17 If the field $\mathbb{k}$ is infinite, then there are a strictly decreasing sequence of integers $1 \leq j_{n-D}<\cdots<j_{1}=k$ and homogeneous polynomials $h_{i, j} \in \mathcal{P}$ such that each of the polynomials $g_{i}=f_{i}+h_{i, i+1} f_{i+1}+\cdots+h_{i, k} f_{k}$ for $i=j_{1}, \ldots, j_{n-D}$ is homogeneous of degree $d_{i}$ and such that $g_{j_{1}}, \ldots, g_{j_{n-D}}$ form a regular sequence in $\mathcal{P}$.

Proof We follow the proof given by Ritscher [43, Lem. 2.81] filling in some missing details. We show by induction that for each $1 \leq r \leq n-D$ there exists a regular sequence $g_{j_{1}}, \ldots, g_{j_{r}}$ and $\operatorname{dim}\left(\left\langle f_{j_{r}}, \ldots, f_{k}\right\rangle\right)=n-r$. For the base case, we take $j_{1}=k$ and $g_{j_{1}}=f_{k} \neq 0$. Obviously, $\operatorname{dim}\left(\left\langle f_{k}\right\rangle\right)=n-1$. For the inductive step, assume that $g_{j_{1}}, \ldots, g_{j_{r}}$ for $r<n-D$ is a regular sequence in $\mathcal{P}$ and $\operatorname{dim}\left(\left\langle f_{j_{r}}, \ldots, f_{k}\right\rangle\right)=n-r$. Let $P=\left\{\mathfrak{p}_{1}, \ldots, \mathfrak{p}_{t}\right\}$ be the set of all associated primes of $\mathcal{J}=\left\langle g_{j_{1}}, \ldots, g_{j_{r}}\right\rangle$. Then, by Macaulay's Unmixedness theorem (see e.g. [9, Thm. 2.1.6]), we have $\operatorname{dim}\left(\mathfrak{p}_{i}\right)=n-r$ for each $i$. Since $\operatorname{dim}(\mathcal{I})=n-D$ and $n-r>D$, there exists an integer $j_{r+1}$ such that $\operatorname{dim}\left(\left\langle f_{j_{r+1}}, \ldots, f_{k}\right\rangle\right) \leq n-(r+1) \geq D$. Let $j_{r+1}$ be the maximum integer with this property.

Consider the $\mathbb{k}$-linear space $S=\prod_{i=j_{r+1}}^{k} \mathbb{k}^{\left({ }^{d_{j_{r+1}}-d_{i}+n-1}\right)}$ and for each integer $\ell=1, \ldots, t$ the subspace

$$
S_{\ell}=\left\{\left(a_{i, \alpha}\right)_{j_{r+1} \leq i \leq k,|\alpha|=d_{j_{r+1}}-d_{i}} \in S \mid \sum_{i=j_{r+1}}^{k} \sum_{|\alpha|=d_{j_{r+1}}-d_{i}} a_{i, \alpha} x^{\alpha} f_{i} \in \mathfrak{p}_{\ell}\right\} .
$$

We claim that $S_{\ell}$ is a proper subspace of $S$ for each $\ell$. For a proof by reductio ad absurdum assume that $S_{\ell}=S$ for some $\ell$. Then we have $x_{j}^{d_{j_{r+1}}-d_{i}} f_{i} \in \mathfrak{p}_{\ell}$ for each $j$ and for each $i=j_{r}+1, \ldots, j_{r+1}$ and hence $f_{j_{r+1}}, \ldots, f_{k} \in \mathfrak{p}_{\ell}$ since $\mathfrak{p}_{\ell}$ is a prime ideal. By construction, the ideal generated by $f_{j_{r+1}}, \ldots, f_{k}$ is of dimension $n-(r+1)$ which yields a contradiction.

Since $\mathbb{k}$ is assumed to be infinite, $S \neq S_{1} \cup \cdots \cup S_{t}$ by elementary linear algebra. Choose a tuple $\left(a_{i, \alpha}\right)_{i, \alpha} \in S \backslash\left(S_{1} \cup \cdots \cup S_{t}\right)$. Then the corresponding polynomial $g_{j_{r+1}}^{\prime}=\sum_{i=j_{r+1}}^{k} \sum_{|\alpha|=d_{j_{r+1}}-d_{i}} a_{i, \alpha} x^{\alpha} f_{i}$ is a non-zero divisor on $\mathcal{P} / \mathcal{J}$. Note that here $f_{j_{r+1}}$ is multiplied only by a constant. We show now that this constant does not vanish. Indeed, otherwise a linear combination of the polynomials $f_{j_{r+1}+1}, \ldots, f_{k}$ was a non-zero divisor on $\mathcal{P} / \mathcal{J}$ implying that the depth of the ideal $\left\langle f_{j_{r+1}+1}, \ldots, f_{k}\right\rangle$ was greater than its dimension $n-r$ which is not possible. Finally, dividing $g_{j_{r+1}}^{\prime}$ by the coefficient of $f_{j_{r+1}}$ yields a new polynomial $g_{j_{r+1}}$ of the desired form to extend our regular sequence.

We note that one obtains a regular sequence for a generic choice of the polynomials $h_{i, j}$. Furthermore, this proposition implies that for a given ideal $\mathcal{I}=\left\langle f_{1}, \ldots, f_{k}\right\rangle$ we may assume w.l.o.g. that $f_{1}, \ldots, f_{n-D}$ is a regular sequence. Since we have $\operatorname{deg}\left(g_{i}\right) \leq \operatorname{deg}\left(f_{i}\right)$ for each $i$, this assumption may only increase the following upper bounds. 
Proposition 4.18 ([35, Cor. 3.5, page 107]) Let $f_{1}, \ldots, f_{k}$ be a regular sequence of homogeneous polynomials and assume that $\mathcal{I}=\left\langle f_{1}, \ldots, f_{k}\right\rangle$ is in Nother position. Then, $\operatorname{deg}(\mathcal{I}, \prec) \leq d_{1}+\cdots+d_{k}-k+1$.

Theorem 4.19 If $d_{k} \geq 2$, then a linear change of variables $\phi$ exists such that the transformed ideal $\phi(\mathcal{I})$ is in Nother position. Furthermore, there are polynomials $h_{i} \in \mathcal{I} \cap \mathbb{k}\left[x_{i}, x_{n-D+1}, \ldots, x_{n}\right]$ for $i=1, \ldots, n-D$ and coefficients $g_{1}, \ldots, g_{k} \in \mathcal{P}$ such that $h_{i}$ is monic in $x_{i}$ and $h_{i}=g_{1} f_{1}+\cdots+g_{k} f_{k}$ with $\operatorname{deg}\left(g_{j} f_{j}\right) \leq 3 d_{1} \cdots d_{n-D}$.

Proof By Prop. 4.17 we may assume that the sequence $f_{1}, \ldots, f_{n-D}$ is regular. Applying Lem. 4.16 provides then polynomials $p_{1}, \ldots, p_{n-D}$ such that their homogenizations form a regular sequence in ${ }^{h} \mathcal{P}$ and satisfy $\operatorname{deg}\left({ }^{h} p_{j}\right) \leq$ $\max \left\{\operatorname{deg}\left({ }^{h} \mathcal{I}_{j-1}\right), \operatorname{deg}\left(f_{j}\right)\right\} .{ }^{h} \mathcal{I}_{j-1}$ is the saturation of the ideal generated by ${ }^{h} f_{1}, \ldots,{ }^{h} f_{j-1}$ w.r.t. $x_{n+1}$ and thus $\operatorname{deg}\left({ }^{h} \mathcal{I}_{j-1}\right) \leq \operatorname{deg}\left(\left\langle{ }^{h} f_{1}, \ldots,{ }^{h} f_{j-1}\right\rangle\right) \leq$ $d_{1} \cdots d_{j-1}$. This observation implies that $\operatorname{deg}\left({ }^{h} p_{1}\right)=d_{1}$ and $\operatorname{deg}\left({ }^{h} p_{j}\right) \leq$ $d_{1} \cdots d_{j-1}$ for each $j>1$. Let $\mathcal{J} \subset{ }^{h} \mathcal{P}$ be the homogeneous ideal generated by the polynomials ${ }^{h} p_{j}$. The Nother normalization lemma asserts the existence of a linear change of variables $\phi$ such that $\phi(\mathcal{J})$ is in Nother position. Since at the end, we will set $x_{n+1}=1$, we choose $\phi$ by ignoring $x_{n+1}$ in the Nœether normalization process which is always possible. It is easy to see that the sequence $\phi\left({ }^{h} p_{1}\right), \ldots, \phi\left({ }^{h} p_{n-D}\right)$ remains regular.

We consider now a degree reverse lexicographic order with $x_{n+1} \prec x_{n} \prec$ $\ldots \prec x_{n-D+1} \prec x_{i}$ and $x_{i} \prec x_{j}$ for $j \neq i$ and $j<n-D$. Since $\phi(\mathcal{J})$ is in Nother position, the reduced Gröbner basis $G$ of $\phi(\mathcal{J})$ for $\prec$ contains a polynomial $w_{i} \in \mathbb{k}\left[x_{i}, x_{n-D+1}, \ldots, x_{n}, x_{n+1}\right]$ which is monic in $x_{i}$. Since $\phi(\mathcal{J})$ is in addition generated by a regular sequence, Prop. 4.18 implies that the degrees of the elements of $G$ is at most $\sum_{j=1}^{n-D}\left(\operatorname{deg}\left({ }^{h} p_{j}\right)-1\right)+1 \leq d_{1}+d_{1}+$ $d_{1} d_{2}+\cdots+d_{1} \cdots d_{n-D-1}$. Using a simple induction and the fact that $2 \leq d_{j}$ for all $j$, we conclude that $d_{1}+d_{1}+d_{1} d_{2}+\cdots+d_{1} \cdots d_{n-D-1} \leq d_{1} \cdots d_{n-D}$. Now, there exist coefficients $a_{j} \in{ }^{h} \mathcal{P}$ such that $w_{i}=a_{1}{ }^{h} p_{1}+\cdots+a_{n-D}{ }^{h} p_{n-D}$. We note that $\left.w_{i}\right|_{x_{n+1}=1} \in \phi(\mathcal{I})$ is a monic polynomial in $x_{i}$ with coefficients in $\mathbb{k}\left[x_{n-D+1}, \ldots, x_{n}\right]$. However, there still remains to find the maximum degree of the representation of this polynomial in terms of the original generators $f_{i}$.

Thus, we next aim at expressing the polynomials ${ }^{h} p_{j}$ in terms of the generators ${ }^{h} f_{1}, \ldots,{ }^{h} f_{n-D}$. We know that ${ }^{h} p_{j}=x_{n+1}^{c_{j}}{ }^{h} f_{j}+q_{j}$ with $q_{j} \in{ }^{h} \mathcal{I}_{j-1}$ and $\operatorname{deg}\left(q_{j}\right) \leq d_{1} \cdots d_{j-1}$. From [49, Lem. 3.18], we deduce that for some exponent $\mu_{j} \leq 2 d_{1} \cdots d_{j-1}$ the product $x_{n+1}^{\mu_{j}} q_{j}$ belongs to the ideal generated by ${ }^{h} f_{1}, \ldots,{ }^{h} f_{j-1}$. This shows that $x_{n+1}^{\mu} w_{i}$ with $\mu=2 d_{1} \cdots d_{n-D}$ can be written as a linear combination of the polynomials ${ }^{h} f_{j}$ and the maximal degree of this expression is at most $3 d_{1} \cdots d_{n-D}$. If we set $h_{i}=\left.x_{n+1}^{\mu} w_{i}\right|_{x_{n+1}=1}$, then the desired conditions hold for $h_{i}$, and this terminates the proof.

Remark 4.20 The new bound given by the above theorem improves the existing results including the bound $d^{n}\left(d^{n}+1\right)$ stated in [13, Sec. 1] where $d=\max \left\{d_{1}, \ldots, d_{k}\right\}$ and also the bound $\left(d_{1} \cdots d_{n-D}\right)^{2}$ from [38, Thm. 10]. 
We now state some consequences of this theorem. Dickenstein et al. 13] applied their variant of Thm. 4.19 to give a bound for the degree w.r.t. only a subset of variables for the membership problem. Following their proof and using our new bound, we obtain the next result.

Proposition 4.21 Assume $d_{k} \geq 2$. A polynomial $f \in \mathcal{P}$ lies in the ideal $\mathcal{I}$, if and only if coefficients $g_{1}, \ldots, g_{k} \in \mathcal{P}$ exist such that $f=g_{1} f_{1}+\cdots+g_{k} f_{k}$ and the degree of each summand $g_{i} f_{i}$ w.r.t. the variables $x_{1}, \ldots, x_{n-D}$ is at most $\max \left\{\operatorname{deg}(f), d_{1}+3(n-D) d_{1} \cdots d_{n-D}\right\}+3 d_{1} \cdots d_{n-D}$.

Consider the element $f=g_{1} f_{1}+\cdots+g_{k} f_{k}$ in the ideal generated by the polynomials $f_{1}, \ldots, f_{k}$ of degrees $d_{1} \geq \cdots \geq d_{k}$. The first upper bound for the coefficients, $\operatorname{deg}\left(g_{i}\right) \leq \operatorname{deg}(f)+2\left(k d_{1}\right)^{2^{n-1}}$, was established by Hermann 26]. We will mimic the proof of [30, Thm. 5] to give a sharper upper bound. We first recall a generic degree bound due to Hermann, see e.g. [37, page 312].

Proposition 4.22 Consider a linear system of equations

$$
\sum_{j=1}^{s} h_{i j} X_{j}=c_{i}, \quad i=1, \ldots, t
$$

with coefficients $h_{i j}, c_{i} \in \mathcal{P}$ which has at least one solution. Then, the system possesses a solution $g_{1}, \ldots, g_{s}$ with $\operatorname{deg}\left(g_{i}\right) \leq c+(d s)^{2^{n}}$ where $d=$ $\max \left\{\operatorname{deg}\left(h_{i j}\right)\right\}$ and $c=\max \left\{\operatorname{deg}\left(c_{i}\right)\right\}$.

Theorem 4.23 Assume $d_{k} \geq 2$. A polynomial $f \in \mathcal{P}$ lies in $\mathcal{I}$, if and only if coefficients $g_{1}, \ldots, g_{k} \in \mathcal{P}$ exits such that $f=g_{1} f_{1}+\cdots+g_{k} f_{k}$ and the degree of each $g_{i}$ is at most $\operatorname{deg}(f)+\left(k d_{1}^{D}\right)^{2^{n-D}}$.

Proof W.l.o.g., we may assume that $\mathcal{I}$ is in Noether position. Any ideal member $f \in \mathcal{I}$ can be written as a linear combination $f=g_{1} f_{1}+\cdots+g_{k} f_{k}$ where the degree of each summand $g_{i} f_{i}$ w.r.t. $x_{1}, \ldots, x_{n-D}$ is at most $B$ with $B$ the bound in Prop. 4.21. Set $\mathcal{P}_{D}=\mathbb{k}\left[x_{n-D+1}, \ldots, x_{n}\right]$. We consider now $g_{i}, f_{i}$ and $f$ as elements of the polynomial ring $\mathcal{P}_{D}\left[x_{1}, \ldots, x_{n-D}\right]$. This leads to representations $g_{i}=\sum_{j} u_{i j} m_{i j}, f_{i}=\sum_{j} w_{i j} m_{i j}^{\prime}$ and $f=\sum_{j} v_{j} m_{j}^{\prime \prime}$ with coefficients $u_{i j}, w_{i j}, v_{j} \in \mathcal{P}_{D}$ and terms $m_{i j}, m_{i j}^{\prime}, m_{j}^{\prime \prime} \in \mathbb{k}\left[x_{1}, \ldots, x_{n-D}\right]$. These satisfy for each $i, j$ the estimates $\operatorname{deg}\left(m_{i j} m_{i j}^{\prime}\right) \leq B, \operatorname{deg}\left(w_{i j}\right) \leq d_{1}$ and $\operatorname{deg}\left(v_{j}\right) \leq \operatorname{deg}(f)$.

Since we look for an upper bound for the degrees of the $g_{i}$, we consider the coefficients $u_{i j}$ as unknowns over $\mathcal{P}_{D}$ and try to bound their degrees using Prop. 4.22, We enter the above representations of $g_{i}, f_{i}$ and $f$ into the linear combination $f=g_{1} f_{1}+\cdots+g_{k} f_{k}$ and extract linear equations for the $u_{i j}$ over $\mathcal{P}_{D}$. By equating the coefficients of each term (in the variables $x_{1}, \ldots, x_{n-D}$ ) of degree at most $B$ on both sides, we derive a linear system of equations over $\mathcal{P}_{D}$. Solving the resulting system yields the coefficients $g_{1}, \ldots, g_{k}$. The number of variables in each linear equation is at most $k$ times the number of terms in $x_{n-D+1}, \ldots, x_{n}$ of degree at most $d_{1}$, i. e. at most $k\left(\begin{array}{c}D+d_{1} \\ D\end{array}\right) \leq k d_{1}^{D}$. Moreover, the right hand side in each linear equation is a polynomial of degree at most $\operatorname{deg}(f)$. Prop. 4.22 implies now the existence of a solution with $\operatorname{deg}\left(g_{i}\right) \leq$ $\operatorname{deg}(f)+\left(k d_{1}^{D}\right)^{2^{n-D}}$ and this yields the desired bound. 
We conclude this section by providing another consequence of Thm. 4.19 Mayr and Ritscher [38, Thm. 36] applied Prop. 4.15 to prove the upper bound

$$
2\left(\frac{1}{2}\left(\left(d_{1} \cdots d_{n-D}\right)^{2(n-D)}+d_{1}\right)\right)^{2^{D}}
$$

for the degrees of the elements of any reduced Gröbner basis of $\mathcal{I}$. In their proof, they exploited that the homogenization of $\mathcal{I}$ contains a homogeneous regular sequence of degree at most $\left(d_{1} \cdots d_{n-D}\right)^{2}$. We now improve this result. The proof of Thm. 4.19 entails that there are polynomials $p_{1}, \ldots, p_{n-D} \in \mathcal{I}$ such that ${ }^{h} p_{1}, \ldots,{ }^{h} p_{n-D}$ is a regular sequence of degree at most $d_{1} \cdots d_{n-D}$, cf. [38, Lem. 35], which yields the following sharper bound.

Corollary 4.24 If $d_{k} \geq 2$, then the degrees of the elements of any reduced Gröbner basis of $\mathcal{I}$ are bounded by

$$
2\left(\frac{1}{2}\left(\left(d_{1} \cdots d_{n-D}\right)^{n-D}+d_{1}\right)\right)^{2^{D}} .
$$

\section{Acknowledgments.}

The authors would like to thank the anonymous reviewers for their helpful comments which helped us to improve the manuscript. The third author received funding from the European Union's Horizon 2020 research and innovation programme under grant agreement No H2020-FETOPEN-2015-CSA 712689

\section{References}

1. D. Bayer and M. Stillman. A criterion for detecting m-regularity. Invent. Math. 87(1), pages 1-11, (1987).

2. D. Bayer and M. Stillman. Computation of Hilbert functions. J. Symb. Comput., 14(4), pages 31-50, (1992).

3. D. Bayer and D. Mumford. What can be computed in algebraic geometry? Computational algebraic geometry and commutative algebra. Cambridge University Press. Symp. Math., 34, pages 1-48, (1993).

4. T. Becker and V. Weispfenning. Gröbner bases: a computational approach to commutative algebra. Springer-Verlag, (1993).

5. I. Bermejo and Ph. Gimenez. Saturation and Catelnuovo-Mumford regularity. J. Algebra, 303, pages 592-617, (2006).

6. A.M. Bigatti and P. Conti and L. Robbiano and C. Traverso. A "divide and conquer" algorithm for Hilbert-Poincaré series, multiplicity and dimension of monomial ideals. Lect. Notes Math., 673, pages 76-88, (1993).

7. W.D. Brownawell. Bounds for the degrees in the Nullstellensatz. Ann. of Math., 126(3), pages 577-591, (1987).

8. W.D. Brownawell and D.W. Masser. Multiplicity estimates for analytic functions. II. Duke Math. J., 47, pages 273-295, (1980).

9. W. Bruns and J. Herzog. Cohen-Macaulay rings. Cambridge University Press, (1998). 
10. B. Buchberger. Ein Algorithmus zum Auffinden der Basiselemente des Restklassenringes nach einem nulldimensionalen Polynomideal. $\mathrm{PhD}$ thesis, Universität Innsbruck, (1965).

11. B. Buchberger. A criterion for detecting unnecessary reductions in the construction of Gröbner bases. In symbolic and algebraic computation (EUROSAM'79, Internat. Sympos., Marseille.) Lecture Notes in Compute. Sci., Springer, Berlin, Volume 72, pages 3-21, (1979)

12. D. Cox and J. Little and D. O'Shea. Ideals, varieties, and algorithms. Springer-Verlag, New York, third edition, (2007).

13. A. Dickenstein and N. Fitchas and M. Giusti and C. Sessa. The membership problem for unmixed polynomial ideals is solvable in single exponential time. Discrete Appl. Math., 33(1-3), pages 73-94, (1991).

14. T. Dubé. The structure of polynomial ideals and Gröbner bases. SIAM J. Comput., 19, pages 750-773, 1990.

15. D. Eisenbud. Commutative Algebra with a View toward Algebraic Geometry. SpringerVerlag, New York, (1995).

16. D. Eisenbud and B. Sturmfels. Finding sparse systems of parameters. J. Pure Appl. Algebra, 94(2), pages 143-157, (1994).

17. S. Eliahou and M. Kervaire. Minimal Resolutions of Some Monomial Ideals. J. Alg. 129, pages 1-25 (1990).

18. R. Fröberg. An introduction to Gröbner bases. John Wiley \& Sons Ltd., Chichester, (1997).

19. A. Galligo. A propos du théorème de preparation de Weierstrass. Lect. Notes Math., 409, pages 543-579, (1974).

20. V.P. Gerdt. and Yu.A. Blinkov. Involutive bases of polynomial ideals. Math. Comput. Simul., 45(5-6), pages 519-541 (1998).

21. M. Giusti. Some effectivity problems in polynomial ideal theory. In EUROSAM 84 (Cambridge, 1984), volume 174 of Lecture Notes in Comput. Sci., pages 159-171. Springer, Berlin, (1984).

22. R. Hartshorne. Algebraic geometry 8th printing. Springer-Verlag, Berlin, (1997).

23. A. Hashemi. Polynomial complexity for Hilbert series of Borel type ideals. Albanian J. Math, 1(3), pages 145-155, (2007).

24. J. Heintz. Definability and fast quantifier elimination in algebraically closed fields. Theor. Comput. Sci., 24, pages 239-277, (1983).

25. J. Herzog and T. Hibi. Monomial Ideals. Graduate Texts in Mathematics 260, SpringerVerlag, London, (2011).

26. G. Hermann. Die Frage der endlich vielen Schritte in der Theorie der Polynomideale. Math. Ann. 95(1), pages 736-788, (1926).

27. M. Janet. Les modules de formes algébriques et la théorie générale des systèmes différentielles. Ann. Sci. Éc. Norm. Supér. 41, pages 27-65, (1924).

28. M. Janet. Leçons sur les Systèmes d'Équations aux Dérivées Partielles. Cahiers Scientifiques, Fascicule IV. Gauthier-Villars, Paris, (1924).

29. Z. Jelonek. On the effective Nullstellensatz. Invent. Math. 162(1), pages 1-17, (2005).

30. M. Kartzer. Computing the dimension of a polynomial ideal and membership in lowdimensional ideals. Master thesis, Technische Universität München, (2008).

31. G. Kemper. A course in commutative algebra. Springer-Verlag, Heidelberg, (2001).

32. J. Kollár. Sharp effective Nullstellensatz. J. Amer. Math. Soc. 1(4), pages 963-975, (1988).

33. D. Lazard. Résolution des systèmes d'équations algébriques. Theoret. Comput. Sci. 15(1), pages 77-110, (1981).

34. D. Lazard. Gröbner bases, Gaussian elimination and resolution of systems of algebraic equations. In Computer algebra (London, 1983), volume 162 of Lecture Notes in Comput. Sci., pages 146-156. Springer, Berlin, (1983).

35. M. Lejeune-Jalabert. Effectivité de calculs polynomiaux. Cours de D.E.A, Institiute Fourier, Grenoble, (1984).

36. D. Masser and G. Wüstholz . Fields of large transcendence degree generated by values of elliptic functions. Invent. Math., 72, pages 407-464, (1983). 
37. E.W. Mayr and A.R. Meyer. The complexity of the word problems for commutative semigroups and polynomial ideals. Adv. in Math., 46(3), pages 305-329, (1982).

38. E.W. Mayr and S. Ritscher. Dimension-dependent bounds for Gröbner bases of polynomial ideals. J. Symb. Comput., 49, pages 78-94, (2013).

39. H.M. Möller and F. Mora. Upper and lower bounds for the degree of Groebner bases. In EUROSAM 84 (Cambridge, 1984), volume 174 of Lecture Notes in Comput. Sci., pages 172-183. Springer, Berlin, (1984).

40. T. Mora. Solving polynomial equation systems II: Macaulay's paradigm and Gröbner technology. Cambridge University Press, (2005).

41. D. Rees. A Basis Theorem for Polynomial Modules. Proc. Cambridge Phil. Soc. 52, pages $12-16,(1956)$.

42. C. Riquier. Les Systèmes d'Équations aux Derivées Partielles. Gauthier-Villars, Paris, (1910).

43. S. Ritscher. Degree bounds and complexity of Gröbner bases of important classes of polynomial ideals. PhD thesis, Technische Universität München, (2012).

44. W.M. Seiler. A combinatorial approach to involution and $\delta$-regularity I: Involutive bases in polynomial algebras of solvable type. Appl. Alg. Eng. Comm. Comp. 20, pages 207-259, (2009).

45. W.M. Seiler. A combinatorial approach to involution and $\delta$-regularity II: Structure analysis of polynomial modules with Pommaret bases. Appl. Alg. Eng. Comm. Comp. 20, pages 261-338, (2009).

46. W.M. Seiler. Involution -The formal theory of differential equations and its applications in computer algebra. Algorithms and Computation in Mathematics, 24, Springer-Verlag, Berlin, (2009).

47. W.M. Seiler. Effective genericity, $\delta$-regularity and strong Noether position. Commun Algebra, 40(10), pages 3933-3949, (2012).

48. R.P. Stanley. Hilbert Functions of Graded Algebras. Adv. Math. 28, pages 57-83, (1978).

49. M. Sombra. A sparse effective Nullstellensatz. Adv. Appl. Math., 22(2), pages 271-295, (1999).

50. G. Valla. Problems and results on Hilbert functions of graded algebras. Prog. Math. 166, pages 293-344, (1998).

51. A.Yu. Zharkov and Yu.A. Blinkov. Involutive approach to investigating polynomial systems. Math. Comput. Simul., 42(4-6), pages 323-332, (1996). 